\title{
Perpendicular magnetic tunnel junctions with multi-interface free layer
}

Pravin Khanal ${ }^{1}$, Bowei Zhou ${ }^{1}$, Magda Andrade ${ }^{1}$, Yanliu Dang ${ }^{2,3}$, Albert Davydov ${ }^{2}$, Ali Habiboglu ${ }^{1}$, Jonah Saidian ${ }^{1}$, Adam Laurie ${ }^{1}$, Jian-Ping Wang ${ }^{4}$, Daniel B Gopman ${ }^{2}$ and Weigang Wang ${ }^{1 *}$

1. Department of Physics, University of Arizona, Tucson, AZ 85721, USA

2. Materials Science \& Engineering Division, National Institute of Standards and Technology, Gaithersburg, MD 20899, USA

3. Department of Electrical \& Computer Engineering, Purdue University, West Lafayette, Indiana 47907, USA

4. Department of Electrical \& Computer Engineering, University of Minnesota, Minneapolis, MN 55455 , USA

Future generations of magnetic random access memory demand magnetic tunnel junctions that can provide simultaneously high magnetoresistance, strong retention, low switching energy and small cell size below $10 \mathrm{~nm}$. Here we study perpendicular magnetic tunnel junctions with composite free layers where multiple ferromagnet/nonmagnet interfaces can contribute to the thermal stability. Different nonmagnetic materials ( $\mathrm{MgO}, \mathrm{Ta}, \mathrm{Mo}$ ) have been employed as the coupling layers in these multi-interface free layers. The evolution of junction properties under different annealing conditions is investigated. A strong dependence of tunneling magnetoresistance on the thickness of the first CoFeB layer has been observed. In junctions where $\mathrm{Mo}$ and $\mathrm{MgO}$ are used as coupling layers, large tunneling magnetoresistance above $200 \%$ has been achieved after $400^{\circ} \mathrm{C}$ annealing. 
Magnetic tunnel junction with perpendicular magnetic anisotropy (pMTJ) is one of the leading candidates for non-volatile magnetic random-access memories (MRAM). ${ }^{1,2}$ Ideally, MRAM cells made of pMTJ should exhibit high tunneling magnetoresistance (TMR $>200 \%$ ), be thermally stable at room temperature (>10 years), occupy only a small footprint $(<10 \mathrm{~nm}$ ), and operate with minimum energy consumption by spin-transfer torques (STT), ${ }^{3,4}$ spin orbit torques (SOT), ${ }^{5}$ voltage controlled magnetic anisotropy (VCMA), ${ }^{6,7}$ or other methods. ${ }^{8-10}$ In particular when the recording layer of a pMTJ is a single ferromagnetic (FM) layer with interfacial PMA, the areal perpendicular energy density is usually $1-2 \mathrm{~mJ} / \mathrm{m}^{2}$, which cannot provide enough retention when the junction size is below $10 \mathrm{~nm} \cdot{ }^{11-14}$ Generally, three types of pMTJ are under investigation to solve this problem. In the first type, FMs with bulk perpendicular magnetic anisotropy, such as FePd,${ }^{15,16}$ or $\mathrm{MnGa},{ }^{17}$ alloys are employed, where the thermal stability factor $(\Delta)$ can be increased by increasing the thickness of the FM layer. However, the TMR in these junctions are typically lower than that of $\mathrm{CoFeB} / \mathrm{MgO}$ due to the lack of coherent tunneling effect. ${ }^{18}$ In the second approach, shape anisotropy is employed to promote the outof-plane easy axis in junctions where the thickness of the CoFeB free layer is much larger than the lateral dimension of the junction. ${ }^{19} \mathrm{~A}$ pMTJ smaller than $3 \mathrm{~nm}$ has been successfully achieved with this approach and STT switching has been demonstrated. ${ }^{20}$ This method, however, requires a thick free layer, which may lead to difficulties in obtaining fast switching and device fabrication. In the third method, multiple CoFeB/non-magnet(NM) interfaces are used to enhance the overall PMA of the free layer, where both the CoFeB and NM are limited to very thin thickness ( $1 \mathrm{~nm})$. Significant increases of $\Delta$ and switching efficiency have been realized when the single CoFeB free layer was replaced by a CoFeB/NM/CoFeB/MgO composite free layer. ${ }^{21-24}$ Further modification of stack structure was used to increase the coupling and thermal stability of the free layer. ${ }^{25,26}$ For example, the performance of the pMTJs with a quadinterface free layer has been shown to be substantially enhanced compared to that of doubleinterface. ${ }^{27}$

In this work, we investigated the transport properties in pMTJs with multi-interface free layers (MIFL), where different NM materials, such as MgO, Ta, and Mo, have been employed as the coupling layers to enhance the TMR. The perpendicular magnetic anisotropy, interlayer magnetic coupling and magnetoresistance can be controlled by varying the FM thickness, NM thickness and post-growth thermal annealing treatment. As a result, a TMR ratio as high as $212 \%$ have been achieved in junctions with MIFLs where three CoFeB layers are coupled through Mo and $\mathrm{MgO}$, which to the best of our knowledge is the highest TMR in this type of pMTJs.

The films in this work were fabricated in a 12-source UHV sputtering system (AJA International) with a base pressure of $10^{-7} \mathrm{~Pa}\left(10^{-9} \mathrm{Torr}\right)$. The structure of the MTJ films is $\mathrm{Si} / \mathrm{SiO}_{2} / \mathrm{Ta}(12$ $\mathrm{nm}) / \mathrm{Ru}(15 \mathrm{~nm}) / \mathrm{Ta}(10 \mathrm{~nm}) / \mathrm{Mo}(0.75 \mathrm{~nm}) / \mathrm{Co}_{20} \mathrm{Fe}_{60} \mathrm{~B}_{20}(1 \mathrm{~nm}) / \mathrm{MgO}(1.5-3.5 \mathrm{~nm}) / \mathrm{MIFL} / \mathrm{Mo}$ (1.9 $\mathrm{nm}) / \mathrm{Ta}(10 \mathrm{~nm}) / \mathrm{Ru}(20 \mathrm{~nm})$. Different MIFL film compositions have been synthesized as detailed below. Circular pMTJs with diameters ranging from $2 \mu \mathrm{m}$ to $100 \mu \mathrm{m}$ were patterned by conventional microfabrication process involving photolithography and ion beam etching, and 
subsequently annealed under varying conditions to be described below. Detailed information on sample fabrication and characterization can be found in our previous publications. $8,12,14,28,29$

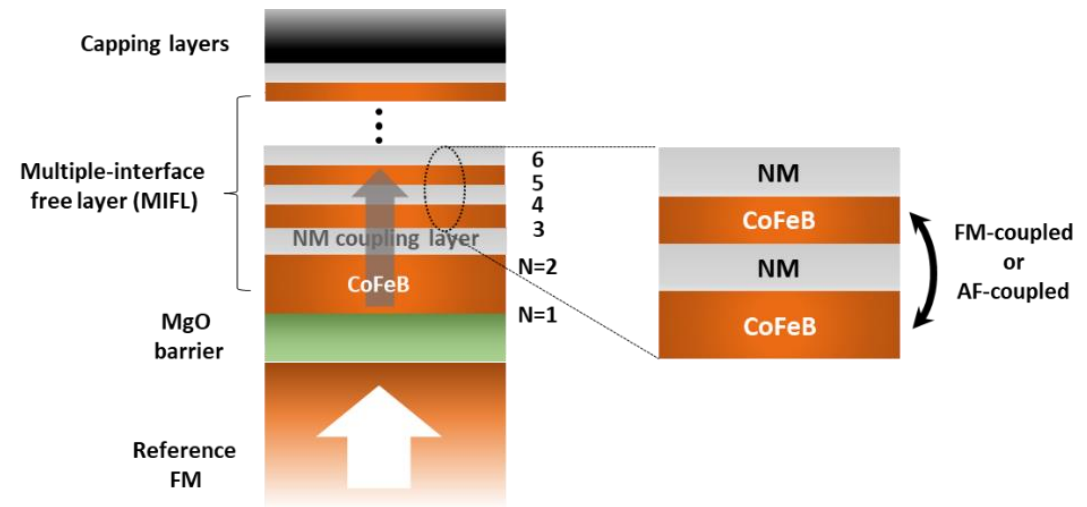

Figure 1. Schematic representation of a pMTJ with multiple-interface free layer (MIFL) where a number of FM layers are coupled through NM layers to function as a single magnetic layer

The schematic structure of a pMTJ with MIFL is shown in Figure 1. Multiple FM layers are coupled through the NM layers to behave like a single free layer of the pMTJ. When FM layers are thin $(\approx 1 \mathrm{~nm})$, the hybridization of $3 d$ orbitals of $F M$ with the $2 p$ orbitals of $O,{ }^{30}$ or with the $5 d$ orbitals of heavy metals, ${ }^{31,32}$ leads to the interfacial PMA. When properly designed, the PMA from each FM/NM interface can add up to each other therefore providing a sufficiently large $\Delta$ for small pMTJ cells. The coupling between the FM layers can be either FM or AF, depending on the needs of a particular application, which can be controlled by the materials and thickness of the FM and NM layers, as well as post-fabrication processes such as thermal annealing. AFcoupled FMs may provide technologically superior performance as it has been predicted that the switching speed can be increased in AF-coupled FMs. ${ }^{33}$ This structure is similar to those used in perpendicular spin valves, ${ }^{34}$ and $\mathrm{pMTJ},{ }^{35}$ except now it combines FM with high spinpolarization with multiple $\mathrm{FM} / \mathrm{NM}$ interfaces that can provide at the same time a large TMR and strong retention after high temperature annealing at $400^{\circ} \mathrm{C}$ or above. CoFeB alloys are a promising candidate for the first FM in the MIFL, owing to the symmetry-conserved tunneling effect at the $\mathrm{Fe} / \mathrm{MgO}$ interface.$^{18}$ However, for the other layers in the MIFL, a combination of different materials could be used to maximize the PMA and interlayer coupling. Ideally, for any pMTJ at a given lateral size, the number of FM layers and therefore the total number of interfaces $(N)$ contributing to PMA energy density could be increased until the desired $\Delta$ is reached.

We first investigated MIFLs with MgO as the coupling layer. In addition to the larger TMR, the $\mathrm{CoFeB} / \mathrm{MgO}$ interface also provides a strong interfacial PMA. ${ }^{36,37}$ Interlayer exchange coupling in epitaxial $\mathrm{Fe} / \mathrm{MgO}$ structures has been observed previously, where AF coupling has been observed when the $\mathrm{MgO}$ thickness is less than $0.8 \mathrm{~nm} .{ }^{38,39}$ However, most of these studies were performed with thick Fe layers where the magnetic easy axis lies within the plane. For 
sputtered $\mathrm{MgO}$ that is sandwiched between two perpendicularly magnetized Co layers, AF coupling was also found even when MgO was as thick as $1.3 \mathrm{~nm} .{ }^{40}$ For the MIFL depicted in Figure 1, it is desirable to have a MgO coupling layer that is thick enough to support a strong coupling, but thin enough to contribute only minimal additional series resistance to the overall resistance of the PMTJ - which may be satisfied if current can conduct across pinholes within the thin MgO layer. The magnetic properties of the MIFL with MgO was first investigated in a sample with the structure of $[\mathrm{CoFeB}(0.75 \mathrm{~nm}) / \mathrm{MgO}(0.8 \mathrm{~nm})]_{3}$ by a vibrating sample magnetometer (VSM). The film exhibits PMA as shown in Figure 2a, where an in-plane anisotropy field larger than $1 \mathrm{~T}(10 \mathrm{kOe})$ can be observed. The $\mathrm{MH}$ loops in the low field region is shown in Figure $2 \mathrm{~b}$, where the remanent magnetic moment is about $20 \mathrm{nA} \cdot \mathrm{m}^{2}$ ( $20 \mu \mathrm{emu}$ ) which is about one-third of the saturation moment $\left(60 \mathrm{nA} \cdot \mathrm{m}^{2}\right)$, indicating the three CoFeB layers are AF-coupled.
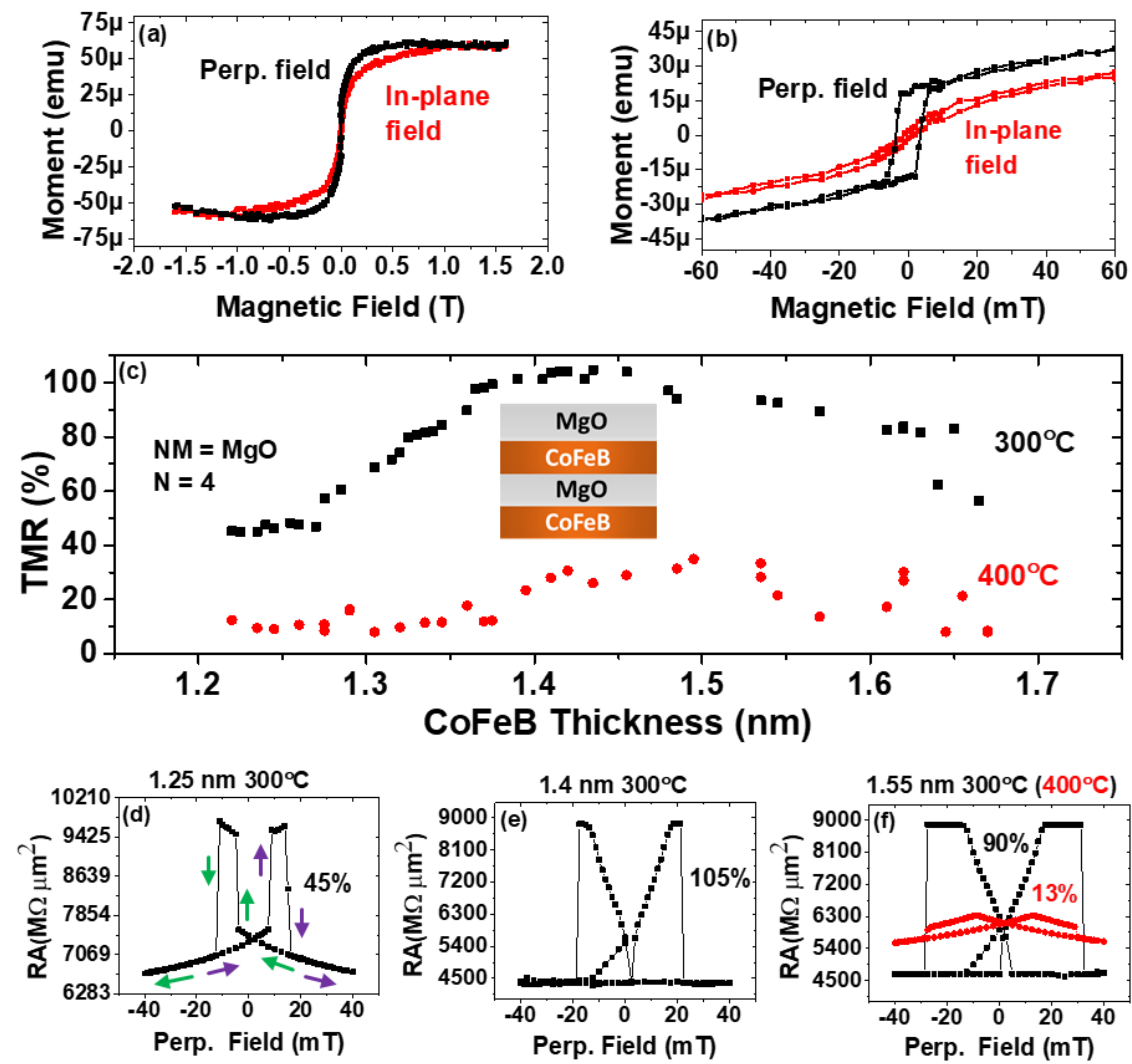

Figure 2. (a) Hysteresis loops of $[\mathrm{CoFeB}(0.75 \mathrm{~nm}) / \mathrm{MgO}(0.8 \mathrm{~nm})]_{\times 3}$ measured under different magnetic fields. (b) The same curves at the low-field region. (c) CoFeB thickness dependence of the TMR in pMTJs with MgO-MIFLs (Inset: schematic of MgO-MIFL). The samples were first annealed at $300^{\circ} \mathrm{C}$ for $10 \mathrm{~min}$, then $400^{\circ} \mathrm{C}$ for another 10 min. (d-f) representative TMR curves of the pMTJs after the $300^{\circ} \mathrm{C}$ annealing. The arrows in (d) show the representative magnetic field sweeping direction [purple (green) towards the positive(negative) field direction]. The red curve in (f) is the TMR of the same sample after the $400^{\circ} \mathrm{C}$ annealing.

Next, pMTJs with MIFLs of the structure of CoFeB $(1.2 \mathrm{~nm}-1.7 \mathrm{~nm}) / \mathrm{MgO}(0.9 \mathrm{~nm}) / \mathrm{CoFeB}(1.3$ $\mathrm{nm}$ ) were fabricated. These pMTJs are denoted as $N=4$ because the total PMA originates from 
three $\mathrm{CoFeB} / \mathrm{MgO}$ interfaces and one $\mathrm{CoFeB} / \mathrm{Mo}$ interface (recall the capping layers are $\mathrm{Mo} / \mathrm{Ta} / \mathrm{Ru}$ ). Since the first CoFeB in the MIFL is the one contributing to both TMR and PMA, it is critical to study the thickness dependence of this layer. The TMR of these junctions after annealing at $300^{\circ} \mathrm{C}$ for $10 \mathrm{~min}$ is plotted in Figure 2c. The TMR is about $45 \%$ when CoFeB is 1.25 $\mathrm{nm}$ thick (Figure $2 \mathrm{~d}$ ), which increases to more than $100 \%$ when CoFeB is $1.45 \mathrm{~nm}$ thick. Further increase of CoFeB thickness beyond this point leads to a slight decrease of TMR (Figure $2 f$ ). Note MTJs located on the edge of the wafer were not included due to poor pattering as a result of edge bead formation. The TMR is, however, reduced across the entire thickness series when the same pMTJs were annealed again (after testing at RT) at $400^{\circ} \mathrm{C}$ for $10 \mathrm{~min}$. A number of processes simultaneously occur during the annealing process, most importantly the formation of the $\mathrm{CoFe}(001) / \mathrm{MgO}(001)$ epitaxial structure with the $\mathrm{B}$ diffusing out of $\mathrm{CoFeB}$, and the reduction of interfacial oxidation which eventually leads to proper hybridization of Oxygen $2 p$ orbitals and $\mathrm{Fe} / \mathrm{Co} 3 d$ orbitals that is required for a strong PMA. Typically the parallel state resistance $\left(R_{\mathrm{P}}\right)$ of the junction momentarily drops at the beginning of the annealing, resulting from the initial establishment of the highly conductive $\Delta_{1}$ channel, followed by a steady increase due to the gradual deterioration of that channel when other atomic species inevitably diffuse into the barrier. ${ }^{41,42}$ Despite the increase of $R_{P}$, the TMR may continue to increase at $400^{\circ} \mathrm{C}$ for up to a few hours of annealing, provided that increases in the anti-parallel state resistance $\left(R_{\mathrm{AP}}\right)$ due to the reduction of the $\Delta_{2}$ and $\Delta_{5}$ conduction channels outpaces of the increase of $R_{\mathrm{P}}{ }^{41,43}$ The comparison of the TMR curves from the same junction after annealing at $300^{\circ} \mathrm{C}$ and $400^{\circ} \mathrm{C}$ is presented in Figure $2 \mathrm{f}$. In addition to the increase of $R_{P}$, we note that $R_{A P}$ is decreased as shown by the red TMR curve. The decrease of $R_{\mathrm{AP}}$ in Figure $2 \mathrm{f}$ is accompanied by the disappearance of the sharp switching in the TMR curve, which is likely due to the reduction of PMA of the MIFL, instead of a more transport-intrinsic reason that is usually only expected when the annealing is much longer. ${ }^{41}$ The reduction of PMA might be a result of Boron aggregation at the $\mathrm{CoFeB} / \mathrm{MgO}$ interface in the absence of any "Boron-absorbing" layer adjacent to the CoFeB layer.

Next, we investigated pMTJs with the MIFL of the structure of CoFeB $(1.2 \mathrm{~nm}-1.7 \mathrm{~nm}) / \mathrm{Ta}(1$ $\mathrm{nm}) / \mathrm{CoFeB}(1 \mathrm{~nm}) / \mathrm{MgO}(0.8 \mathrm{~nm})$, which is denoted by $N=4$ with Ta in Figure 3 since now there are two $\mathrm{CoFeB} / \mathrm{MgO}$ interfaces and two $\mathrm{CoFeB} / \mathrm{Ta}$ interfaces that each contribute to PMA. This structure is similar to what was used previously, ${ }^{21-24}$ except here we chose the Ta at $1 \mathrm{~nm}$. Maximum AF-coupling was observed in Co/Ta superlattices when Ta is near $0.7 \mathrm{~nm} .{ }^{44}$ For $\mathrm{CoFeB} / \mathrm{Ta} / \mathrm{CoFeB}$, a sizable AF coupling was obtained when the thickness of Ta is around $1 \mathrm{~nm},{ }^{45}$ which is in agreement with our VSM results shown in Figures $3 \mathrm{a}$ and $3 \mathrm{~b}$. After the $300^{\circ} \mathrm{C}$ annealing for $10 \mathrm{~min}$, maximal TMR of $135 \%$ was obtained as shown in Figure 3c, which is considerably better in those shown in Figure 2. Reasonably high TMR (>100 \%) is present in pMTJs across a wide range of CoFeB thickness. A representative TMR curve under this annealing condition is shown in Figure $3 d$, with sharp transitions between states and a clear AP state. This improved TMR behavior is likely related to the fact that the Ta coupling layer more readily absorbs Boron diffusing out from the CoFeB layer, compared to a MgO coupling layer. 
Subsequent annealing of these pMTJs at $400^{\circ} \mathrm{C}$ for 10 min substantially increased the maximum TMR to above $180 \%$ as shown in Figure $3 f$, which is noticeably higher than previous reports. ${ }^{21-}$ ${ }^{24}$ However, the TMR starts to fall off after the CoFeB thickness exceeds $1.5 \mathrm{~nm}$ and exhibits large fluctuations when CoFeB is thicker than $1.6 \mathrm{~nm}$. This reduction in the TMR is attributed to the loss of the AP state as shown in Figure 3g. Usually, the strength and sign of the interlayer coupling is sensitively depended on the thickness of the FM and NM layers. ${ }^{40,46}$ Here another complexity is involved, which is the PMA of MIFL. Due to the relatively small formation energy between $\mathrm{Ta}$ and $\mathrm{Fe}$, it is known that the PMA of $\mathrm{MgO} / \mathrm{CoFeB} / \mathrm{Ta}$ is not stable when annealed at $400^{\circ} \mathrm{C}^{12}$ which leads to the deterioration of PMA of the MIFL stack. Further annealing of these pMTJs at $500^{\circ} \mathrm{C}$ leads to a dramatic reduction of TMR to nearly zero, consistent with previous studies. ${ }^{12}$
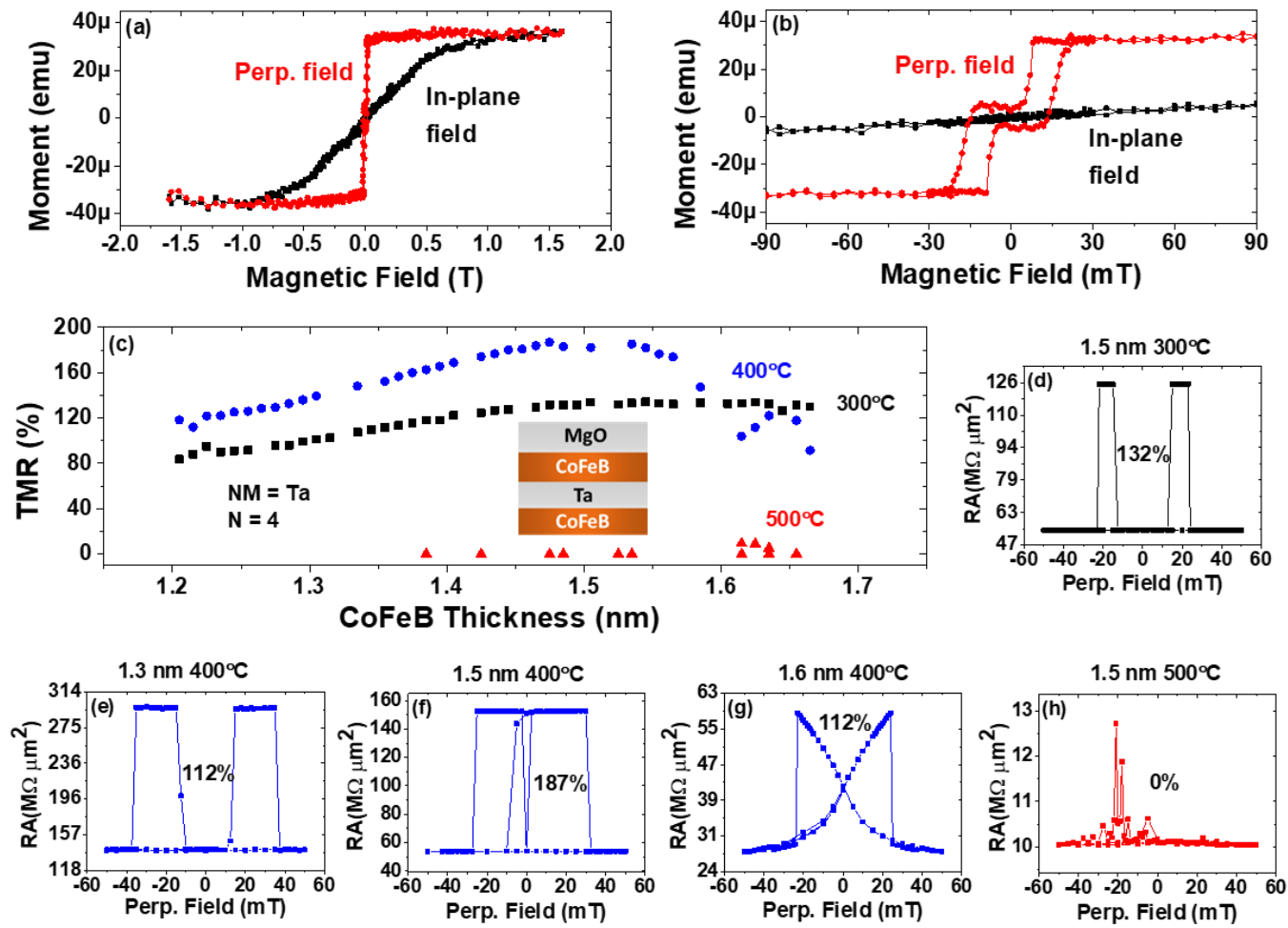

Figure 3. (a) Hysteresis loops of $\mathrm{MgO} / \mathrm{CoFeB} / \mathrm{Ta}(1 \mathrm{~nm}) / \mathrm{CoFeB} / \mathrm{MgO}$ measured on different magnetic fields. (b) The same curves at low field-region. (c) CoFeB thickness dependence of the TMR in PMTJs with Ta-MIFLs (Inset: schematic of Ta-MIFL). The samples were successive annealed at each temperature for $10 \mathrm{~min}$. (d) TMR curve of the $1.5 \mathrm{~nm}$ sample after the $300^{\circ} \mathrm{C}$ annealing. (e-g) TMR curves of three pMTJs after the $400^{\circ} \mathrm{C}$ annealing. (h) TMR of the $1.5 \mathrm{~nm}$ sample after the $500^{\circ} \mathrm{C}$ annealing.

It has been shown that pMTJs with Mo as the heavy metal layer exhibited much higher TMR than that of $\mathrm{Ta}^{12,14,47}$. Therefore, MIFLs with Mo as the coupling layer may provide larger TMR as well. Another benefit of Mo is that its interlayer exchange coupling energy is larger compared to that of $\mathrm{Ta} .{ }^{44}$ It was also shown that Mo can substantially enhance damping. ${ }^{48}$ pMTJs with three CoFeB layers in the MIFL have been fabricated. The MIFL stack structure is CoFeB $(1.2 \mathrm{~nm}-1.7 \mathrm{~nm}) / \mathrm{Mo}(0.9 \mathrm{~nm}) / \mathrm{CoFeB}(1 \mathrm{~nm}) / \mathrm{MgO}(0.9 \mathrm{~nm}) /$ CoFeB $(1.3 \mathrm{~nm})$. These 
samples are denoted as $N=6$ with Mo as plotted in Figure 4. The VSM results show the strong AF coupling for Mo(0.9nm) are presented in Figures $4 \mathrm{a}$ and $4 \mathrm{~b}$. The TMR ratios of the samples after annealing at $300^{\circ} \mathrm{C}$ for $10 \mathrm{~min}$ (red curve) are presented in Figure 4c. The maximum TMR in pMTJs with Mo-MIFL is similar to that of Ta-MIFL under this annealing condition. However, the TMR starts to decay in pMTJs with Mo-MIFL when CoFeB is thicker than $1.5 \mathrm{~nm}$. By comparison, the TMR with Ta-MIFL gains a slight increase over $1.5 \mathrm{~nm}$ to $1.6 \mathrm{~nm}$ under the same annealing condition as shown in Figure 3c. This feature of Mo-MIFL becomes more pronounced after the annealing at $400^{\circ} \mathrm{C}$ for $10 \mathrm{~min}$ as shown in the black curve of Figure $4 \mathrm{c}$, where TMR drops sharply when CoFeB is thicker than $1.4 \mathrm{~nm}$. When the pMTJs are annealed at $400^{\circ} \mathrm{C}$ for one hour, the overall TMR has been increased (blue curve), with maximum TMR reaching $212 \%$ as shown in Figure $4 \mathrm{e}$, which is even higher than the TMR in pMTJs we obtained previously with a single CoFeB layer as the free layer. ${ }^{14}$ However, TMR quickly drops when CoFeB thickness exceeds $1.42 \mathrm{~nm}$. The reduction of TMR is again related to the disappearance of the AP state as shown in the Figure 4f, which is likely due to the loss of PMA of the MIFL. These results highlight the very sensitive dependence of TMR on the first CoFeB layer thickness in the MIFL. The AF coupling peak with Mo varies in different reports, ranging from $0.5 \mathrm{~nm},{ }^{44}$ to $0.8 \mathrm{~nm} .{ }^{49} \mathrm{In}$ another series of pMTJ where the Mo in the MIFL is slightly thicker $(1 \mathrm{~nm})$, a more pronounced AF coupling of the free layer can be seen as shown in the inset of Figure 4c. The free layer switching fields are obviously not symmetric about the zero magnetic field, which is a signature of the AF coupling of the CoFeB in the MIFL. The behavior of these pMTJs is plotted in the green curve in Figure 4c. Interestingly, the range of CoFeB thicknesses where high TMR ratio is observed is extended by nearly $0.1 \mathrm{~nm}$, as evident from the shift of the green curve relative to the black one. 

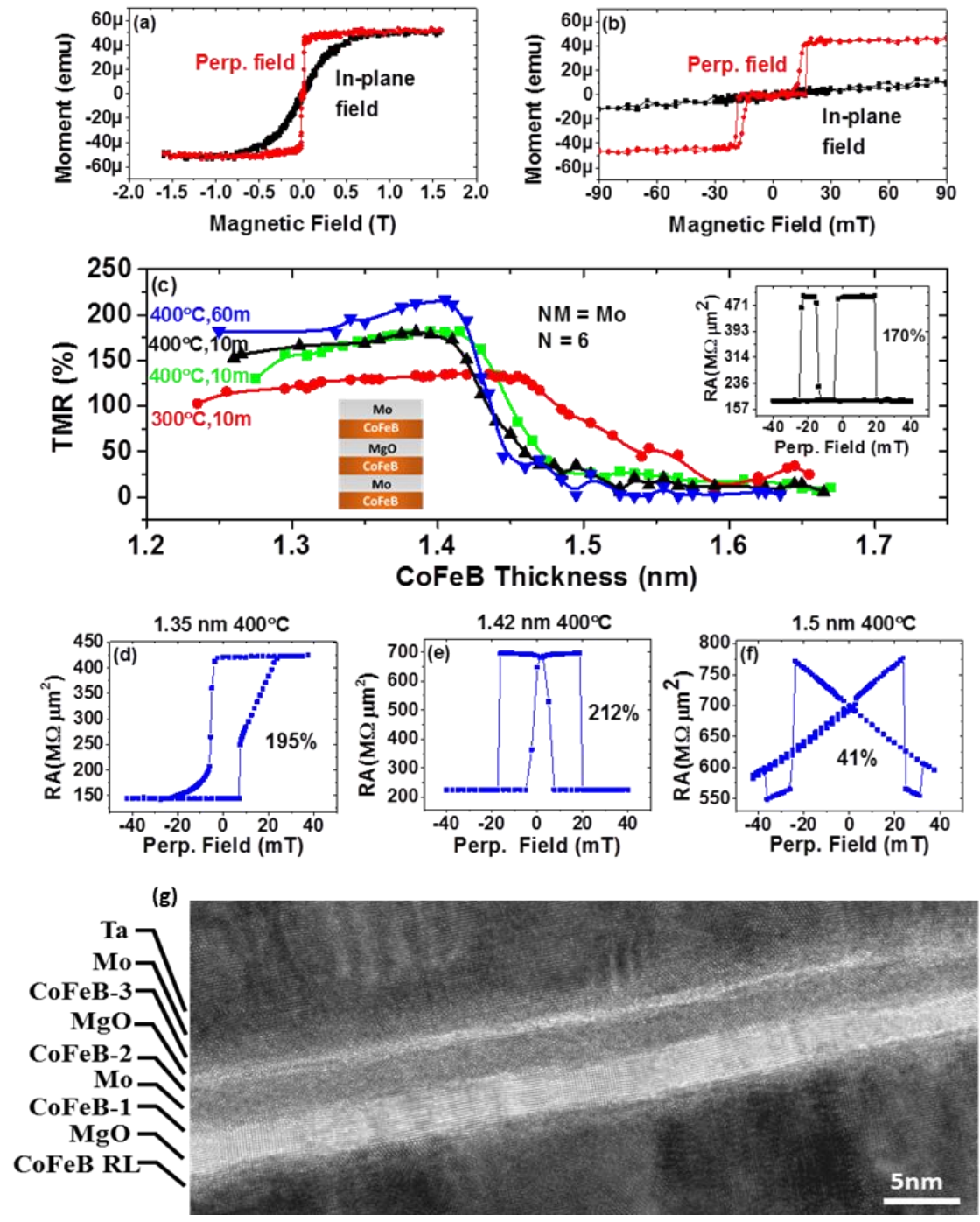

Figure 4. (a) Hysteresis loops of $\mathrm{MgO} / \mathrm{CoFeB} / \mathrm{Mo}(0.9 \mathrm{~nm}) / \mathrm{CoFeB} / \mathrm{MgO} / \mathrm{CoFeB} / \mathrm{Mo}$ measured on different magnetic fields. (b) The same curves at low field-region. (c) CoFeB thickness dependence of the TMR in pMTJs with MoMIFLs (Inset: schematic of Mo-MIFL). The samples with Mo-0.9nm coupling layer were successive annealed at $300^{\circ} \mathrm{C}$ for $10 \mathrm{~min}$ (red dots), $400^{\circ} \mathrm{C}$ for $10 \mathrm{~min}$ (black up-pointing triangles), then $400^{\circ} \mathrm{C}$ for another $50 \mathrm{~min}$ (blue down-pointing triangles, where the total annealing time at $400^{\circ} \mathrm{C}$ is $60 \mathrm{~min}$ ). The green square data points are the TMR values of the samples with the Mo-1nm coupling layer, annealed at $400^{\circ} \mathrm{C}$ for $10 \mathrm{~min}$. The lines are for guiding eyes only. Inset shows the TMR curve of a pMTJ with the Mo- $1 \mathrm{~nm}$ coupling layer. (d-f) Representative TMR curves of three pMTJ after the $400^{\circ} \mathrm{C}$ annealing for 60min. (g) HRTEM image of the pMTJ with Mo-MIFL.

The microstructure of pMTJ with Mo(0.6nm) -MIFL was investigated by TEM and is presented in Figure 4g. The MgO tunnel barrier exhibits good (001) crystalline structure throughout the specimen. The CoFeB reference layer beneath the $\mathrm{MgO}$ tunnel barrier and the first CoFeB in the MIFL show predominantly (001) texture, indicating successful solid-state-epitaxy from the MgO barrier outward during the $400^{\circ} \mathrm{C}$ anneal. The successful recrystallization of the $\mathrm{CoFeB} / \mathrm{MgO} / \mathrm{CoFeB}$ complex is critical for the high TMR ratios observed within these pMTJs. The second and the third CoFeB layers in the MIFL, however, are only partially crystallized. The MgO 
layer in the MIFL is mostly continuous and exhibits partial crystallization from the thermal processing. Note in MIFLs the amorphous second (and third) CoFeB and some pinholes in the $\mathrm{MgO}$ could potentially be advantageous, as they may help to reduce the Gilbert damping and the resistance-area product of the devices, respectively. Note for Ta-MIFL with $\mathrm{N}=6$, the maximum TMR is only $180 \%$, again demonstrating the advantage of Mo as a better HM layer.

These results suggest the first CoFeB thickness must be precisely controlled in order to achieve the largest TMR in pMTJs with MIFL. Though the drop of TMR after a certain threshold thickness of the first CoFeB is a common feature observed in all three types of MIFLs in this study, the decay in PMTJs with Mo-MIFL is most pronounced. Obviously, this phenomenon is related to the reduced PMA of the first CoFeB layer when its thickness is getting larger. However the fast drop of TMR with Mo-MIFL cannot solely be explained by PMA, since PMA is usually stronger in pMTJs with Mo compared to those with Ta. ${ }^{12}$ The presence of interlayer coupling makes the situation more complicated, which can certainly have a large influence on the switching behavior of the CoFeB layers in the MIFL. In particular, the coupling strength may vary with the thickness of the FM layer, which in the Bruno model is due to the Fabry-Perottype interferences of the electron wave functions through multiple reflections in FM layers. ${ }^{46}$ Meanwhile, the magnetic properties of the CoFeB itself at a given thickness is under constant change (such as crystallization and redistribution of $O$ at the $\mathrm{MgO} / \mathrm{CoFeB}$ interface) when annealed at different conditions, which will in turn impact the interlayer magnetic coupling. A more detailed study is needed to understand the evolution of interlayer coupling in these pMTJs. The difference of the two sets of Mo-MIFL samples $(0.9 \mathrm{~nm}$ vs $1 \mathrm{~nm})$ also indicates the range of CoFeB thickness that gives rise to high TMR may be expanded if the AF coupling is enhanced.

To conclude, we have developed PMTJs with MIFL that can potentially simultaneously afford high TMR, strong retention and efficient switching for MRAM cells that are scaled down to small dimensions. Different nonmagnetic materials have been explored as the coupling layer in the MIFL. The TMR of the PMTJ exhibits a strong dependence on the thickness of the first CoFeB layer. Large TMR above $200 \%$ has been achieved after $400^{\circ} \mathrm{C}$ annealing in pMTJs where three CoFeB layers are incorporated in the MIFL.

\section{Acknowledgements}

This work was supported in part by Semiconductor Research Corporation through the Logic and Memory Devices program (Global Research Collaboration), by DARPA through the ERI program (FRANC), and by NSF through DMR-1905783. M.A. and A.L. were supported by the REU supplement of NSF ECCS-1554011.

\section{Data availability}


The data that support the findings of this study are available from the corresponding author upon reasonable request.

\section{References}

1. Yuasa, S., Hono, K., Hu, G. \& Worledge, D. C. Materials for spin-transfer-torque magnetoresistive random-access memory. MRS Bull. 43, 352-357 (2018).

2. Apalkov, D., Dieny, B. \& Slaughter, J. M. Magnetoresistive Random Access Memory. Proc. IEEE 104, 1796-1830 (2016).

3. Ralph, D. C. \& Stiles, M. D. Spin transfer torques. J. Magn. Magn. Mater. 320, 1190-1216 (2008).

4. Brataas, A., Kent, A. D. \& Ohno, H. Current-induced torques in magnetic materials. Nat. Mater. 11, 372-81 (2012).

5. Manchon, A., Železný, J., Miron, I. M., Jungwirth, T., Sinova, J., Thiaville, A., Garello, K. \& Gambardella, P. Current-induced spin-orbit torques in ferromagnetic and antiferromagnetic systems. Rev. Mod. Phys. 91, 035004 (2019).

6. Matsukura, F., Tokura, Y. \& Ohno, H. Control of magnetism by electric fields. Nat. Nanotechnol. 10, 209-220 (2015).

7. Wang, W.-G., Li, M., Hageman, S. \& Chien, C. L. Electric-field-assisted switching in magnetic tunnel junctions. Nat. Mater. 11, 64-8 (2011).

8. Newhouse-Illige, T., Liu, Y., Xu, M., Reifsnyder Hickey, D., Kundu, A., Almasi, H., Bi, C., Wang, X., Freeland, J. W., Keavney, D. J., Sun, C. J., Xu, Y. H., Rosales, M., Cheng, X. M., Zhang, S., Mkhoyan, K. A., Wang, W. G., Hickey, D. R., Kundu, A., Almasi, H., Bi, C., Wang, X., Freeland, J. W., Keavney, D. J., Sun, C. J., Xu, Y. H., Rosales, M., Cheng, X. M., Zhang, S., Mkhoyan, K. A. \& Wang, W. G. Voltage-controlled interlayer coupling in perpendicularly magnetized magnetic tunnel junctions. Nat. Commun. 8, 15232 (2017).

9. He, X., Wang, Y., Wu, N., Caruso, A. N., Vescovo, E., Belashchenko, K. D., Dowben, P. a \& Binek, C. Robust isothermal electric control of exchange bias at room temperature. Nat. Mater. 9, 579-85 (2010).

10. Bi, C., Sun, C., Xu, M., Newhouse-Illige, T., Voyles, P. M. \& Wang, W. Electrical Control of Metallic Heavy-Metal-Ferromagnet Interfacial States. Phys. Rev. Appl. 8, 034003 (2017).

11. Dieny, B. \& Chshiev, M. Perpendicular magnetic anisotropy at transition metal/oxide interfaces and applications. Rev. Mod. Phys. 89, 025008 (2017).

12. Almasi, H., Hickey, D. R., Newhouse-Illige, T., Xu, M., Rosales, M. R., Nahar, S., Held, J. T., Mkhoyan, K. A. \& Wang, W. G. Enhanced tunneling magnetoresistance and perpendicular magnetic anisotropy in $\mathrm{Mo} / \mathrm{CoFeB} / \mathrm{MgO}$ magnetic tunnel junctions. Appl. Phys. Lett. 106, 182406 (2015). 
13. Almasi, H., Sun, C. L., Li, X., Newhouse-Illige, T., Bi, C., Price, K. C., Nahar, S., Grezes, C., Hu, Q., Khalili Amiri, P., Wang, K. L., Voyles, P. M. \& Wang, W. G. Perpendicular magnetic tunnel junction with W seed and capping layers. J. Appl. Phys. 121, 153902 (2017).

14. Almasi, H., Xu, M., Xu, Y., Newhouse-Illige, T. \& Wang, W. G. Effect of Mo insertion layers on the magnetoresistance and perpendicular magnetic anisotropy in $\mathrm{Ta} / \mathrm{CoFeB} / \mathrm{MgO}$ junctions. Appl. Phys. Lett. 109, 032401 (2016).

15. Zhang, D. L., Sun, C., Lv, Y., Schliep, K. B., Zhao, Z., Chen, J. Y., Voyles, P. M. \& Wang, J. P. L10 Fe-Pd Synthetic Antiferromagnet through an fcc Ru Spacer Utilized for Perpendicular Magnetic Tunnel Junctions. Phys. Rev. Appl. 9, 044028 (2018).

16. Zhang, D. L., Schliep, K. B., Wu, R. J., Quarterman, P., Reifsnyder Hickey, D., Lv, Y., Chao, X., Li, H., Chen, J. Y., Zhao, Z., Jamali, M., Mkhoyan, K. A. \& Wang, J. P. Enhancement of tunneling magnetoresistance by inserting a diffusion barrier in L10-FePd perpendicular magnetic tunnel junctions. Appl. Phys. Lett. 112, 152401 (2018).

17. Kubota, T., Ma, Q. L., Mizukami, S., Zhang, X. M., Naganuma, H., Oogane, M., Ando, Y. \& Miyazaki, T. Magnetic tunnel junctions of perpendicularly magnetized L10$\mathrm{MnGa} / \mathrm{Fe} / \mathrm{MgO} / \mathrm{CoFe}$ structures: Fe-layer-thickness dependences of magnetoresistance effect and tunnelling conductance spectra. J. Phys. D. Appl. Phys. 46, 155001(2013).

18. Butler, W. H., Zhang, X.-G., Schulthess, T. C. \& MacLaren, J. M. Spin-dependent tunneling conductance of Fe/MgO/Fe sandwiches. Phys. Rev. B 63, 054416 (2001).

19. Watanabe, K., Jinnai, B., Fukami, S., Sato, H. \& Ohno, H. Shape anisotropy revisited in single-digit nanometer magnetic tunnel junctions. Nat. Commun. 9, 663 (2018).

20. Jinnai, B., Igarashi, J., Watanabe, K., Funatsu, T., Sato, H., Fukami, S. \& Ohno, H. Highperformance shape-anisotropy magnetic tunnel junctions down to $2.3 \mathrm{~nm}$. Tech. Dig. Int. Electron Devices Meet. IEDM 2020-Decem, 24.6.1-24.6.4 (2020).

21. Sato, H., Yamanouchi, M., Ikeda, S., Fukami, S., Matsukura, F. \& Ohno, H. Perpendicularanisotropy CoFeB-MgO magnetic tunnel junctions with a $\mathrm{MgO} / \mathrm{CoFeB} / \mathrm{Ta} / \mathrm{CoFeB} / \mathrm{MgO}$ recording structure. Appl. Phys. Lett. 101, 022414 (2012).

22. Sato, H., Enobio, E. C. I., Yamanouchi, M., Ikeda, S., Fukami, S., Kanai, S., Matsukura, F. \& Ohno, $\mathrm{H}$. Properties of magnetic tunnel junctions with a $\mathrm{MgO} / \mathrm{CoFeB} / \mathrm{Ta} / \mathrm{CoFeB} / \mathrm{MgO}$ recording structure down to junction diameter of $11 \mathrm{~nm}$. Appl. Phys. Lett. 105, 062403 (2014).

23. Lee, S.-E., Baek, J.-U. \& Park, J.-G. Highly Enhanced TMR Ratio and $\Delta$ for Double MgObased p-MTJ Spin-Valves with Top Co2Fe6B2 Free Layer by Nanoscale-thick Iron Diffusion-barrier. Sci. Rep. 7, 11907 (2017).

24. Kim, J. H., Lee, J. Bin, An, G. G., Yang, S. M., Chung, W. S., Park, H. S. \& Hong, J. P. Ultrathin $W$ space layer-enabled thermal stability enhancement in a perpendicular MgO/CoFeB/W/CoFeB/MgO recording frame. Sci. Rep. 5, 16903 (2015). 
25. Chatterjee, J., Chavent, A., Fettar, F., Auffret, S., Ducruet, C., Joumard, I., Vila, L., Sousa, R. C., Prejbeanu, L. \& Dieny, B. Reduced Thermal Variation of Perpendicular Magnetic Anisotropy in Magnetically Stiffened Dual-W Composite Storage Layer for Spin-TransferTorque Magnetic Random-Access Memory. Phys. Rev. Appl. 12, 4 (2019).

26. Nishioka, K., Honjo, H., Naganuma, H., Nguyen, T. V. A., Yasuhira, M., Ikeda, S. \& Endoh, T. Enhancement of magnetic coupling and magnetic anisotropy in MTJs with multiple CoFeB / MgO interfaces for high thermal stability Enhancement of magnetic coupling and magnetic anisotropy in MTJs with multiple CoFeB / MgO interfaces for high thermal stabili. AIP Adv. 11, 025231 (2021).

27. Nishioka, K., Sato, H., Endoh, T., Honjo, H., Ikeda, S., Watanabe, T., Miura, S., Inoue, H., Tanigawa, T., Noguchi, Y. \& Yasuhira, M. Novel Quad-Interface MTJ Technology and its First Demonstration with High Thermal Stability Factor and Switching Efficiency for STTMRAM beyond 2X nm. IEEE Trans. Electron Devices 67, 995-1000 (2020).

28. Newhouse-Illige, T., Xu, Y. H., Liu, Y. H., Huang, S., Kato, H., Bi, C., Xu, M., Leroy, B. . \& Wang, W. G. Temperature dependence of interlayer coupling in perpendicular magnetic tunnel junctions with GdOx barriers. Appl. Phys. Lett. 112, (2018).

29. Xu, M., Li, M., Khanal, P., Habiboglu, A., Insana, B., Xiong, Y., Peterson, T., Myers, J. C., Ortega, D., Qu, H., Chien, C. L., Zhang, W., Wang, J. P. \& Wang, W. G. Voltage-Controlled Antiferromagnetism in Magnetic Tunnel Junctions. Phys. Rev. Lett. 124, 187701 (2020).

30. Yang, H. X., Chshiev, M., Dieny, B., Lee, J. H., Manchon, A. \& Shin, K. H. First-principles investigation of the very large perpendicular magnetic anisotropy at $\mathrm{Fe} / \mathrm{MgO}$ and Co|MgO interfaces. Phys. Rev. B 84, 054401 (2011).

31. Bruno, P. Tight-binding approach to the orbital magnetic moment and magnetocrystalline anisotropy of transition-metal monolayers. Phys. Rev. B 39, 865(R) (1989).

32. Weller, D., Stohr, J., Nakajima, R., Carl, A., Samant, M. G., Chappert, C., Beauvillain, P., Veillet, P. \& Held, G. A. Microscopic Origin of Magnetic Anisotropy in Au/Co/Au Probed with X-Ray Magnetic Circular Dichroism. Phys. Rev. Lett. 75, 3752-3755 (1995).

33. Camsari, K. Y., Pervaiz, A. Z., Faria, R., Marinero, E. E. \& Datta, S. Ultrafast Spin-TransferTorque Switching of Synthetic Ferrimagnets. IEEE Magn. Lett. 1-1 (2016).

34. Mangin, S., Ravelosona, D., Katine, J. A., Carey, M. J., Terris, B. D. \& Fullerton, E. E. Current-induced magnetization reversal in nanopillars with perpendicular anisotropy. Nat. Mater. 5, 210-215 (2006).

35. Liu, E., Swerts, J., Couet, S., Mertens, S., Tomczak, Y., Lin, T., Spampinato, V., Franquet, A., Van Elshocht, S., Kar, G., Furnemont, A. \& De Boeck, J. [Co/Ni]-CoFeB hybrid free layer stack materials for high density magnetic random access memory applications. Appl. Phys. Lett. 108, 132405 (2016).

36. Ikeda, S., Miura, K., Yamamoto, H., Mizunuma, K., Gan, H. D., Endo, M., Kanai, S., 
Hayakawa, J., Matsukura, F. \& Ohno, H. A perpendicular-anisotropy CoFeB-MgO magnetic tunnel junction. Nat. Mater. 9, 721-724 (2010).

37. Worledge, D. C., Hu, G., Abraham, D. W., Sun, J. Z., Trouilloud, P. L., Nowak, J., Brown, S., Gaidis, M. C., O'Sullivan, E. J. \& Robertazzi, R. P. Spin torque switching of perpendicular Ta|CoFeB|MgO-based magnetic tunnel junctions. Appl. Phys. Lett. 98, 022501 (2011).

38. Faure-Vincent, J., Tiusan, C., Bellouard, C., Popova, E., Hehn, M., Montaigne, F. \& Schuhl, a. Interlayer Magnetic Coupling Interactions of Two Ferromagnetic Layers by Spin Polarized Tunneling. Phys. Rev. Lett. 89, 107206 (2002).

39. Katayama, T., Yuasa, S., Velev, J., Zhuravlev, M. Y., Jaswal, S. S. \& Tsymbal, E. Y. Interlayer exchange coupling in Fe/MgO/Fe magnetic tunnel junctions. Appl. Phys. Lett. 89, 112503 (2006).

40. Nistor, L. E., Rodmacq, B., Auffret, S., Schuhl, A., Chshiev, M. \& Dieny, B. Oscillatory interlayer exchange coupling in $\mathrm{MgO}$ tunnel junctions with perpendicular magnetic anisotropy. Phys. Rev. B 81, 220407R (2010).

41. Wang, W. G., Ni, C., Miao, G. X., Weiland, C., Shah, L. R., Fan, X., Parson, P., Jordan-sweet, J., Kou, X. M., Zhang, Y. P., Stearrett, R., Nowak, E. R., Opila, R., Moodera, J. S. \& Xiao, J. $\mathrm{Q}$. Understanding tunneling magnetoresistance during thermal annealing in $\mathrm{MgO}$-based junctions with CoFeB electrodes. Phys. Rev. B 81, 144406 (2010).

42. Wang, W. G., Jordan-Sweet, J., Miao, G. X., Ni, C., Rumaiz, A., Shah, L., Fan, X., Parson, P., Stearrett, R., Nowak, R., Moodera, J. S. \& Xiao, J. Q. In situ characterization of rapid crystallization of amorphous CoFeB electrodes in $\mathrm{CoFeB} / \mathrm{MgO} / \mathrm{CoFeB}$ junctions during thermal annealing. Appl. Phys. Lett. 95, 242501 (2009).

43. Wang, W.-G., Hageman, S., Li, M., Huang, S., Kou, X., Fan, X., Xiao, J. Q. \& Chien, C. L. Rapid thermal annealing study of magnetoresistance and perpendicular anisotropy in magnetic tunnel junctions based on MgO and CoFeB. Appl. Phys. Lett. 99, 102502 (2011).

44. Parkin, S. S. P. Systematic variation of the strength and oscillation period of indirect magnetic exchange coupling through the $3 \mathrm{~d}, 4 \mathrm{~d}$, and $5 \mathrm{~d}$ transition metals. Phys. Rev. Lett. 67, 3598-3601 (1991).

45. Cheng, C. W., Cheng, T. I., Shiue, C. H., Weng, C. L., Tsai, Y. C. \& Chern, G. Synthetic antiferromagnetic $\mathrm{MgO} / \mathrm{CoFeB} / \mathrm{Ta}(\mathrm{x}) / \mathrm{CoFeB} / \mathrm{MgO}$ structures with perpendicular magnetic anisotropy. IEEE Trans. Magn. 49, 4433-4436 (2013).

46. Bruno, P. Theory of interlayer magnetic coupling. Phys. Rev. B 52, 411 (1995).

47. Liu, T., Zhang, Y., Cai, J. W. \& Pan, H. Y. Thermally robust Mo/CoFeB/MgO trilayers with strong perpendicular magnetic anisotropy. Sci. Rep. 4, 5895 (2014).

48. Husain, S., Kumar, A., Barwal, V., Behera, N., Akansel, S., Svedlindh, P. \& Chaudhary, S. Spin pumping in ion-beam sputtered C o2FeAl/Mo bilayers: Interfacial Gilbert damping. Phys. Rev. B 97, 064420 (2018). 
49. Zhang, X., Zhang, Y. \& Cai, J. W. Antiferromagnetically coupled perpendicular magnetic anisotropic CoFeB/MgO films across a Mo spacer with high thermal stability. J. Appl. Phys. 118, 143903 (2015). 